\title{
Bestrahlungserfolg bei Knochenmetastasen
}

Bei Patienten mit ossären Metastasen steht der Erhalt der Lebensqualität im Vordergrund. Eine internationale Studie zeigt, dass sich der Effekt einer Strahlentherapie mit zwei speziellen Lebensqualitäts-Scores gut abbilden lässt.

ollegen der European Organisation for Research and Treatment of Cancer (EORTC) nahmen in ihre Studie 79 Patienten mit schmerzhaften Knochenmetastasen auf (mittleres Alter 63 Jahre, Karnofsky-Index 70). In den meisten Fällen handelte es sich um metastasierte Brust-, Prostata- oder Lungenkarzinome. Vor der Radiotherapie sowie einen Monat danach wurden sie mit dem auf Knochenmetastasen zugeschnittenen Fragebogen QLQ-BM22 befragt. Er erfasst die Lokalisation betroffener Körperareale, Schmerzcharakteristika, Verände- rungen des Aktivitätsniveaus („funktionelle Interferenz") sowie psychosoziale Aspekte. Gleichzeitig wurde der QLQC30 angewendet, der Aktivitätsmöglichkeiten in verschiedenen Bereichen sowie tumorspezifische Symptome misst.

Von 59 auswertbaren Patienten war in 22 Fällen ein partielles Ansprechen auf die Strahlentherapie mit Reduktion der Schmerzen oder der Opiatdosierung dokumentiert. Bei acht Patienten kam es zur Schmerzprogression und in 29 Fällen konnte keine klare Veränderung nachgewiesen werden.
Bei den Patienten, die angesprochen hatten, verbesserten sich im QLQ-BM22 die Bereiche Schmerzlokalisation (-22,7 Punkte), Schmerzcharakteristik $(-36,4)$ und funktionelle Interferenz $(+30,9)$ gegenüber denen, die nicht ansprachen, jeweils signifikant. Gleiches galt für die Domänen körperliche Aktivität $(+16,7)$, Rollenfunktion $(+20,5)$ und Schmerz $(-40,9)$ im QLQ-C30.

Fazit: Der Radiotherapieerfolg bei Knochenmetastasen ist nicht nur durch Schmerzskalen oder Opiatreduktion, sondern auch zwei Lebensqualitäts-Fragebögen valide prüfbar. Andreas Fischer

Zeng $L$ et al. Quality of life after palliative radiation therapy for patients with painful bone metastases: Results of an international study validating the EORTC-BM22. Int J Radiat Oncol Biol Phys. 2012;84(3):e337-42.

\section{Pädiatrische Palliativmedizin: Eltern und Kliniker finden unterschiedliche Dinge wichtig}

\section{Eines von fünf Kindern mit einer malignen Erkrankung stirbt daran. Eine standardisierte Befragung sollte klären, ob Kernelemente der palliativmedizi- nischen NCP-Leitlinien (National Consensus Project, USA) auch in der Pädiatrie von betroffenen Eltern und Klinikern als wichtig eingeschätzt werden.}

V validierte Fragebögen wurden an 140 Eltern gesendet, die ihre Kinder zwischen 2005 und 2011 verloren hatten; 75 (54\%) davon antworteten. Ihre Beurteilungen wurden mit denen von 48 Angestellten der pädiatrischen Onkologie verglichen (Antwortrate $91 \%$ ). Dabei handelte es sich um Ärzte (57\%), Krankenschwestern (34\%) sowie Sozialarbeiter $(10 \%)$ des größten Kinderkrankenhauses in Toronto, ON/Kanada. Die 20 Kernelemente der NCP-Leitlinien konnten mit sehr wichtig, wichtig, neutral, nicht sehr wichtig oder gar nicht wichtig beurteilt werden.

Die Bedeutung von 15 der 20 Kernelemente wurde von Eltern wie von den in der pädiatrischen Onkologie Tätigen als hoch eingeschätzt (d.h. > 60\% der beiden Gruppen beurteilten sie als wichtig). Die höchsten Bewertungen erhielten: Beteiligung eines speziellen Palliativversorgungsteams, telefonischer Zugang zu einem Palliativspezialisten rund um die
Uhr, Möglichkeit zur direkten Klinikaufnahme, Vorbereitung der Eltern auf medizinische Aspekte des Sterbeprozesses, elterliche Entscheidung, ob das Kind zuhause oder in der Klinik sterben darf.

Im Vergleich maßen die Eltern der Gabe einer Antitumor-Therapie im letzten Lebensmonat des Kindes $(\mathrm{p}<0,01)$ und dem Hinzuziehen eines spirituellen Mentors $(\mathrm{p}<0,03)$ größere Bedeutung $\mathrm{zu}$ als die Kliniker. Die insgesamt hohe Bewertung der späten Antitumor-Therapie durch die Eltern geht wahrscheinlich auf ihr Bedürfnis zurück, nichts unversucht zu lassen. Eltern, deren Kinder eine solche Behandlung im letzten Lebensmonat erhalten hatten, erachteten diesen Punkt nicht als wichtig.

Von den als wichtig eingeschätzten 15 Kernelementen standen nach Meinung von Klinikern und Eltern nur drei überwiegend (> $60 \%$ der Zeit) zur Verfügung. Der Zugang zu folgenden wichtigen Elementen war am wenigsten wahrschein-

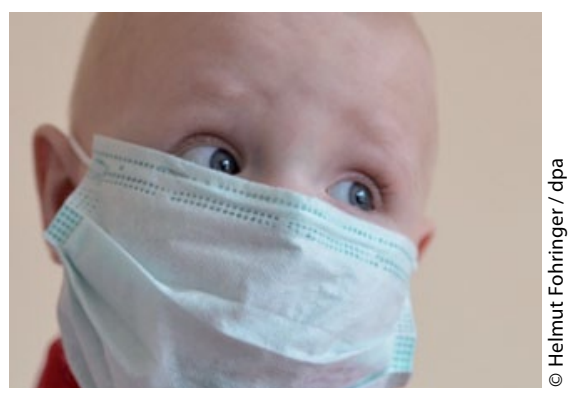

lich: eine direkte Aufnahme in die Klinik, die Unterstützung der Geschwister des erkrankten Kindes und die Vorbereitung der Eltern auf medizinische Aspekte des eigentlichen Sterbeprozesses.

Fazit: Die Mehrzahl der Hauptelemente der NCP-Guidelines zur Palliativversorgung wird von den betroffenen Eltern und den in der pädiatrischen Onkologie Tätigen übereinstimmend als wichtig erachtet. Dessen ungeachtet stehen diese Kernelemente Kindern mit fortgeschrittenen malignen Erkrankungen nicht uneingeschränkt zur Verfügung. Die Gründe hierfür zu analysieren, wäre der erste Schritt für eine Abhilfe, so die Autoren.

Brigitte Schalhorn

Kassam A et al. Moving toward quality palliative cancer care: Parent and clinician perspectives on gaps between what matters and what is accessible. J Clin Oncol. 2013;31(7):910-5. 